\title{
Unique challenges in the management of a Giant retroperitoneal Schwannoma associated with vertebral body destruction and spinal canal invasion
}

Mubarak A. Algahtany, MD, FRCSC, Ahmad M. Almalki, MD, SB-Surg, Ismaeel D. Albeshre, MD, Rabab N. Badri, MD.

\begin{abstract}
الأورام الشفانية الخلف صفاقيه نادره بينما العملاق منها والمصاحَب بانحلال

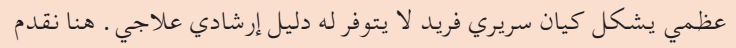

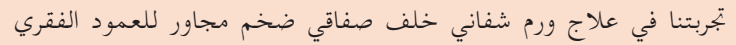

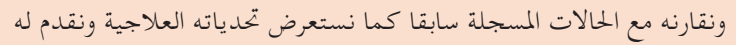

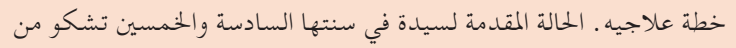

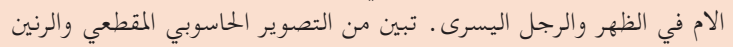

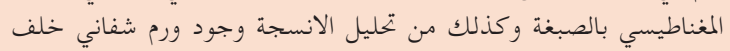

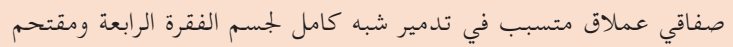

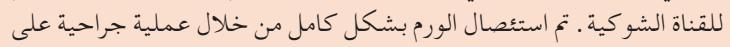

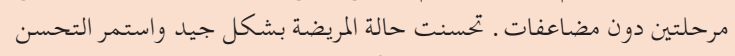

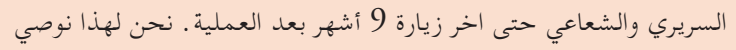

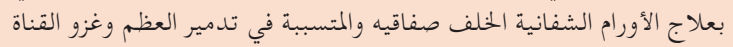

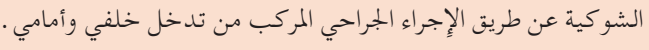

Retroperitoneal schwannomas are rare, and giant lesions associated with osteolysis are unique clinical entities for which management guidelines are lacking. Herein, we present our experience with treating a large paraspinal retroperitoneal schwannoma, compare it with previously reported cases, highlight the challenges faced with its management, and propose a treatment plan. A 56-year-old female patient presented with back and left leg radicular pains. Contrast-enhanced CT and MRI scanning and histological analysis confirmed the presence of a giant retroperitoneal schwannoma causing near-complete destruction of the fourth lumbar vertebral body and spinal canal invasion. The tumor was totally removed by a two-step operation with no adverse consequences. The patient recovered well and remained in good clinical and radiological status 9 months post-surgery. Therefore, retroperitoneal schwannomas causing bone destruction and spinal canal invasion are best treated through a combined posterior-anterior approach.

Neurosciences 2021; Vol. 26 (3): 289-294 doi: 10.17712/nsj.2021.3.20200184

From the Division of Neurosurgery (Algahtany), Department of
Surgery, College of Medicine, King Khalid University, from the Department of Surgery (Almalki), Department of Neurosurgery (Albshre), Department of Histopathology (Badri), Asir Central Hospital, Abha, Kingdom of Saudi Arabia

Received 23rd December 2020. Accepted 28th April 2021.

Address correspondence and reprint request to: Dr. Mubarak A. Algahtany, Division of Neurosurgery, Department of Surgery, College of Medicine, King Khalid University, Abha, Kingdom of Saudi Arabia E-mail: mbalgahtany@kku.edu.sa

ORCID: https://orcid.org/0000-0001-8524-9769

Cchwannomas in the retroperitoneal space are rare; Sthey account for less than 3\% of all schwannomas. ${ }^{1}$ Retroperitoneal schwannomas (RSs) are often benign (99\%) and giant-sized because the expansile retroperitoneal space allows the tumor to enlarge even before presenting; therefore, one-third of RS cases are incidentally discovered. ${ }^{2,3}$ The presenting symptoms are usually nonspecific: abdominal pain and distension are the most typical presenting features followed by back pain and radicular leg symptoms. ${ }^{2,4}$

Paraspinal retroperitoneal schwannomas that erode the spinal column are exceedingly rare with only four cases previously reported in the recent 15-year English literature. ${ }^{1,3-5}$ They are unique clinical entities because of their rarity, presenting features, and complexity of management. The challenges in managing RS include ensuring a correct preoperative diagnosis, achieving an oncologic resection with no vascular or neurological injury, and maintaining the spinal column stability postoperatively. Therefore, the surgical resection of sizable RS poses a tremendous challenge and effective surgical management guidelines are lacking.

Disclosure. The authors declare no conflicting interests, support or funding from any drug company. 
In this paper, we present our management strategy for a massive RS that caused almost the complete lysis of the fourth lumbar (L4) vertebral body resulting in spinal canal invasion with severe neural compression. We propose a practical surgical management plan based on the lessons learned from this case and our updated review of previously reported cases.

Case Report. Patient information and clinical presentation. A previously healthy 56-year-old female patient presented to another hospital with back pain and stabbing pain near the left knee for nine months. She had no abdominal symptoms of any kind or changes in her sphincter functions. She had no personal or family history of neurofibromatosis.

Investigation with computed tomography (CT) and magnetic resonance imaging (MRI) revealed a giant retroperitoneal tumor that had caused over $90 \%$ of the L4 body and left facet destruction, spinal canal invasion, and severe thecal sac compression. In the index hospital, to manage what was presumed to be a malignant neoplasm, the patient underwent an L4 laminectomy, limited tumor removal from the spinal canal, and posterior fixation from the third to the fifth lumbar vertebra (L3-L5). Postoperatively, the patient developed numbness in the left leg besides continuing to have the preoperative symptoms. The patient was then referred to our hospital for further management following a postoperative pathological diagnosis of benign schwannoma.

Clinical findings. Upon presentation to our center, 3 months following the index surgery, she still complained of back pain, left leg radicular pain, and numbness along the L4 dermatome. Physical examination revealed a palpable firm mass filling the left lower abdomen and hypoesthesia in the left L4 dermatome. She had a weak left knee extension and diminished left knee reflex.

Diagnostic assessment. Routine laboratory studies showed no clinically significant abnormalities. Contrastenhanced CT and MRI scanning revealed a solid enhancing intraabdominal, paravertebral tumor of size $11 \times 9 \times 11$ centimeter $(\mathrm{cm})$ filling the left retroperitoneal space and causing near-complete lysis of L4 vertebral body, left pedicle, facet, and transverse process. The tumor extended into the spinal canal causing severe neural compression (Figure 1). The tumor infiltrated the left psoas muscle and was inseparable from it. It compressed and displaced the left renal vein, aorta, inferior vena cava, and left common iliac vessels. It had also compressed and displaced the left kidney superiorly and the left ureter anteriorly, causing dilatation of its pelvicalyceal part. We reviewed the pathological specimen from the index hospital and confirmed the histological diagnosis of Grade 1 schwannoma as per the World Health Organization (WHO) classification.

Therapeutic intervention. A two-step operation was

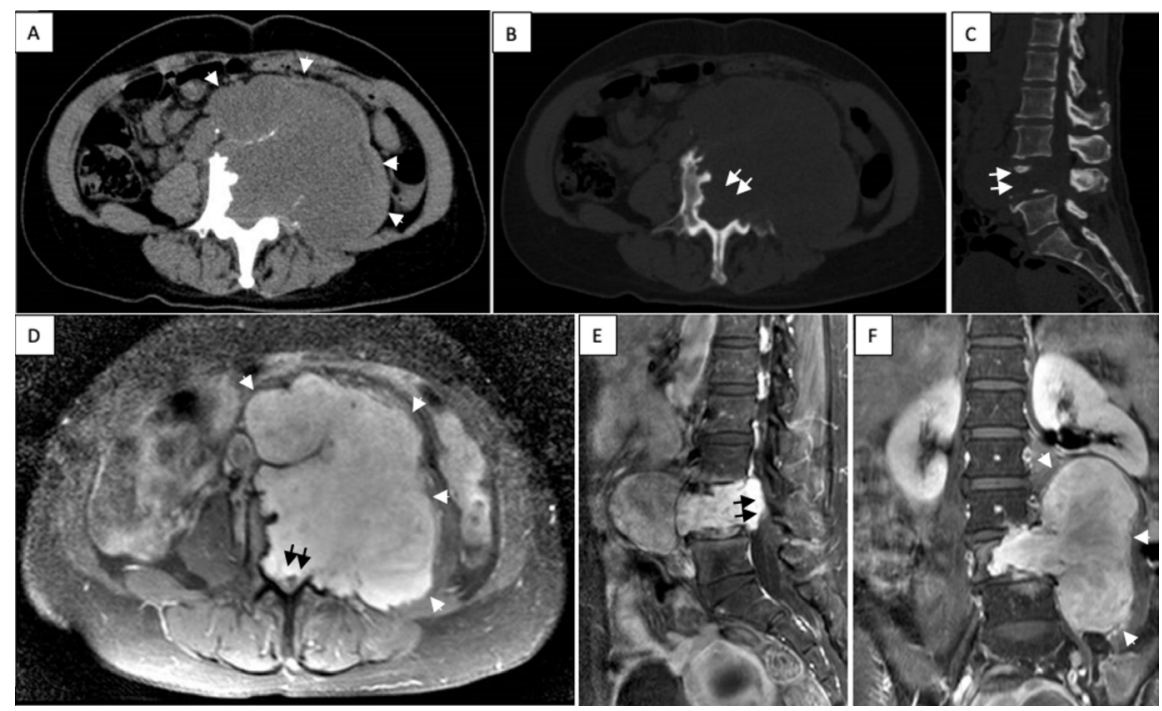

Figure 1 - Preoperative CT and MRI scan images demonstrate the extent of the retroperitoneal tumor (single white arrows), L4 destruction (double white arrows), and spinal canal invasion (double black arrows). A-C) Plain CT scan; A) Soft tissue and B) bone window axial images of the abdomen at the L4 pedicle level, C) bone window sagittal image of the lumbar spine. D-F) Contrast-enhanced T1-weighted MRI scan images of the lumbar spine; D) Axial with fat saturation, E) sagittal, and F) coronal. 


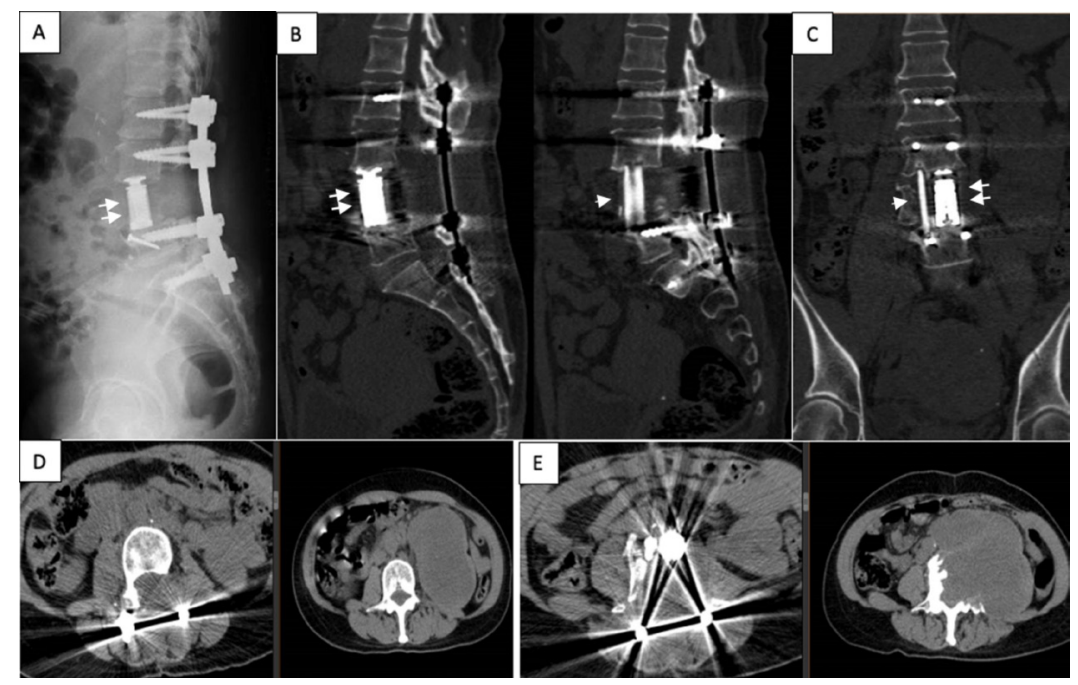

Figure 2 - Postoperative 6 months follow-up, plain x-ray A) and CT scan images B-E) demonstrate a good fusion of the cage (double white arrows) and the fibular graft (single white arrows) A-C), as well as total tumor resection and lack of recurrence (D and E). A) standing lateral radiograph of the lumbar spine, B) sagittal, C) coronal bone window images. D) Axial soft tissue window image at the L3 pedicle level, E) at the L4 pedicle level with preoperative control images on the right side.

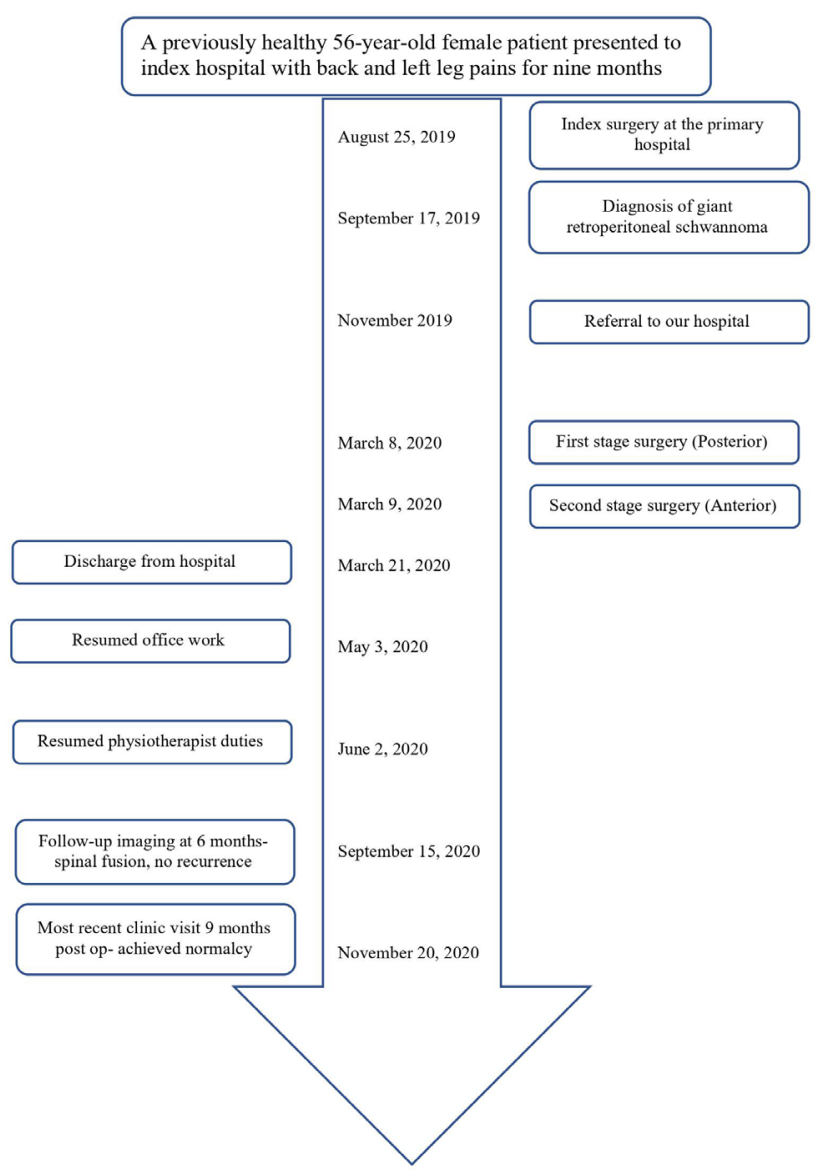

Figure 3 - Timeline of the presented case. 


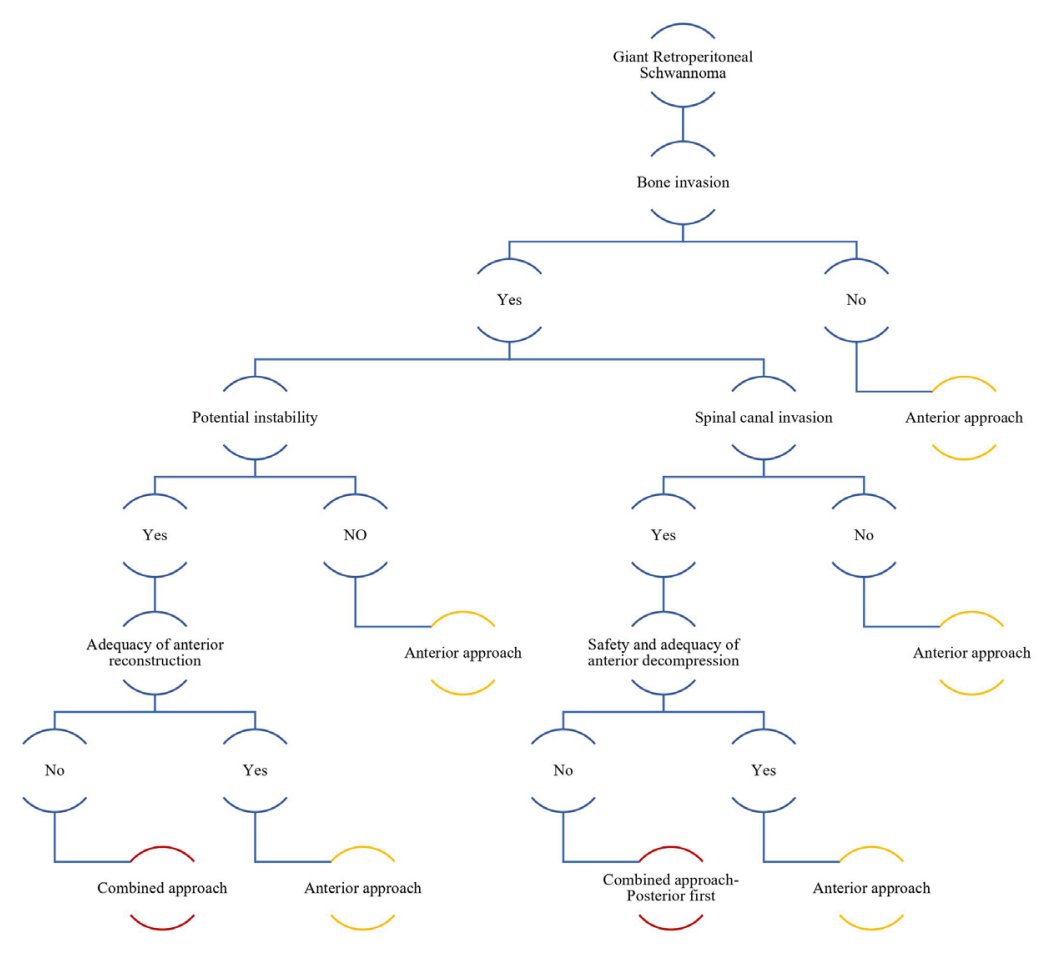

Figure 4 - A flow chart for choosing the most appropriate surgical approach.

performed because of the tumor's location and large size, L4 destruction, spinal canal invasion, and severe vascular and neural compression. The procedure was performed by a multidisciplinary team including spine surgeons and a surgical oncologist.

The first stage of the surgery was by an expanded posterior approach in the prone position. During this stage, the residual parts of L4 laminae were removed along with bilateral facetectomy and right pedicle subtraction. The tumor was dissected from the left L4 nerve root, mostly preserving its integrity with only one incidental durotomy that was subsequently microscopically repaired. The ventral aspect of the dura was freed while being dissected bilaterally from the tumor, using the extended posterolateral approach. This was followed by an instrumented posterior fusion from the second lumbar to the first sacral vertebra (L2-S1).

The second stage of the surgery was performed the following day using a midline transabdominal transperitoneal approach in the supine position. During this stage, the tumor was dissected from the left kidney, left ureter, and displaced abdominal vessels.
The iliolumbar vein, specifically, was adherent to the inferior pole of the tumor, which made dissection difficult. Following the circumferential dissection of the tumor, it was amputated at the spot of invasion into the L4 body. The osseous involvement was gradually but entirely disengaged and the spinal canal was completely decompressed. No vascular, neural, or dural injuries occurred. The estimated blood loss was 700 milliliters. To fix the vertebral defect, the L4 body was reconstructed using an expandable titanium cage filled with bone allograft. The reconstruction was augmented with a fibular allograft that was telescoped into a pre-drilled hole at the L3 lower endplate and fixed to the L 5 body with a screw.

Macroscopically, the specimen was well-capsulated, firm, gray in color, and of size $14 \times 9 \times 12 \mathrm{~cm}$. Histopathological analysis revealed a benign WHO Grade 1 schwannoma composed of spindle cells with wavy nuclei that displayed occasional nuclear palisading. It was composed of mostly hypercellular (Antoni A) areas mixed with hypocellular (Antoni B) areas. The tumor cells strongly and diffusely expressed the S-100 
protein but did not express the epithelial membrane antigen (EMA) or cluster of differentiation 34 (CD34). Mitotic activity, observed by the proliferation index (Ki-67) staining, was rare.

Follow-up and outcome. The patient had an uneventful postoperative course with no general or neurological complications. The back and left leg radicular pains gradually reduced, and the left leg numbness and weakness improved. The patient was satisfied with the outcomes of surgery and resumed her work as a physiotherapist. Radiological imaging performed at six months post-surgery showed stable spinal fusion and absence of tumor recurrence (Figure 2). Her left leg reached normalcy nine months post-operation (Figure 3).

Discussion. Retroperitoneal schwannoma is rare. The benign nature of the neoplasm, lack of specific symptoms, and capacious retroperitoneal space allow the tumor to attain a massive size before presenting clinically. The median size at the time of diagnosis is approximately $15 \mathrm{~cm}^{2}$

Although the majority of RSs are closely associated with the spine, bone erosion is exceedingly rare. ${ }^{3}$ Our review of the recent 15-year English literature revealed only four cases of RS with vertebral bone lysis. ${ }^{1,3-5}$ However, this subgroup of RS forms a unique clinical entity due to its rarity, aggressive radiological features, difficult preoperative diagnosis, and complexity in management. Through this case report and evaluation of similar cases reported in the literature, we aim to clarify this entity, address unique challenges associated with its management, and propose a surgical treatment plan.

Precise diagnosis of RS preoperatively is a significant issue. There are no symptoms or diagnostic features on ultrasound, CT, or MRI specific to RS; this limits preoperative diagnosis and less than $20 \%$ of cases are accurately identified. ${ }^{2,3}$ Furthermore, a core needle biopsy can be non-diagnostic or even misleading towards a malignant neoplasm, especially in cystic and ancient schwannomas. ${ }^{4,6}$ The aggressive features of bone eroding RS, including, large tumor sizes, distortions of myofascial planes, and invasions of bone and spinal canal, are some of the fundamental characteristics of malignant neoplasms. In our case, the initial management team was misled by the aggressive features of the tumor and handled the case as an unresectable malignant neoplasm. Similar cases of misdiagnosis even with preoperative biopsy data have been reported. ${ }^{4}$ However, we still believe that preoperative biopsies are essential adjuncts for the proper diagnosis and management of $\mathrm{RS}$ s. A biopsy-aided accurate preoperative diagnosis will prevent treating the patient as a case of malignant neoplasm based on the aggressive imaging features and allow better planning of curative surgeries.

The surgical management of large RSs with bone and spinal canal invasion requires careful and meticulous planning to aim for an oncologic cure while incurring nil to minimal adverse consequences. This necessitates a multidisciplinary team approach. A capable anesthetic team prepared to deal with intraoperative complications, such as excessive bleeding and pulmonary embolism, is of utmost importance. An adequate amount of packed red blood cells and fresh frozen plasma should be available. Besides, the myriad risks, including root sacrifice, neurological deficit, fertility loss, blood transfusion, retroperitoneal structural injuries, incomplete resection, and need for staging, emphasize the necessity of wellinformed preoperative surgical consent.

The team is encouraged to utilize intraoperative tools, such as a nerve stimulator to identify the nerve of origin and pulse oximeter to continuously monitor the oxygen saturation in the lower extremities. Intraoperative histological analysis of the tumor, through frozen sections, is based on the uncertainty of diagnosis, competency of the histopathology lab, and likelihood of changing the surgical plan according to results from analyzing the sections.

The advantages of piecemeal and en bloc resection techniques are debatable. ${ }^{4}$ Given the benign nature of RS, we believe that a personalized decision should be taken to assure maximum safety; compromising the level of cure (high cure achieved with en bloc technique) is acceptable. Based on conclusions derived from the literature, the size of the tumor, presence and extent of neural canal involvement, bone erosion, potential spinal instability, and the degree of distortion of the abdominal vessels are major deciding factors in deciding the best surgical approach.

Total tumor resection seems to be more beneficial in controlling local recurrence - occurs in up to $20 \%$ of cases following partial resection. ${ }^{1,6}$ The spinal canal and bone marrows are likely places with residuals that may be best addressed using combined approaches, allowing $360^{\circ}$ visualization of the thecal sac.

Nerve root injuries are documented in half of the RSs that manifested spinal canal invasion. ${ }^{1,3,4}$ The surgical approach must be tailored to avoid neural injuries. Whenever the spinal canal, dura, and nerve roots are significantly involved with a tumor, we recommend adopting a combined posterior-anterior approach that allows circumferential dissection of the 
neural elements from the tumor. In our case, the initial posterior approach allowed circumferential untethering of the neural elements, isolation of the nerve root, and easy repair of the incidental durotomy. With this accomplished, the subsequent anterior approach was more straightforward from a neurological perspective and the tumor could be easily mobilized.

The extent of bone erosion and potential postoperative spinal instability are other major deciding factors for the surgical approach. The spinal column is best reconstructed from the front when the vertebral body is significantly deconstructed. The need for posterior augmentation depends on the extent of spinal instability incurred and the previous need for a posterior approach to decompress the spinal canal. In our case, a posterior instrumented fusion was performed during stage 1 (the posterior approach) to address the significant spinal instability incurred due to the destruction of the vertebral body and left facet joint by the tumor and iatrogenic destruction of the right facet during right facetectomy performed to untether the dura.

Vascular injury is another substantial issue during the surgical resection of RS. The tumor adhesion to and displacement of the large abdominal vessels, requiring a great deal of dissection, was documented in all the reported giant RS cases. Vascular control at the early stages of surgery is crucial to prevent vascular injury and consequent severe bleeding that occurs in up to $10 \%$ of surgically removed RSs. ${ }^{1,6}$ The anterior approach undoubtedly gave the best access to and control of retroperitoneal vascular and other structures, therefore, was employed as the second step in our case.

To choose the best surgical approach, we propose a management scheme considering the factors mentioned above (Figure 4). The flowchart suggests that most cases can be managed with an anterior approach. Advances in laparoscopic surgery have allowed endoscopic removal of RSs; ${ }^{6}$ however, none of the endoscopically treated cases had significant bone or spinal canal invasion. The extrapolation of the endoscopic technique to our unique entity has not yet been reported.

Patient perspective. My back and leg pains significantly reduced after the surgery and gradually waned. Therefore, I am completely satisfied with the outcomes of the surgery. I already resumed my work as a physiotherapist.

Conclusion. Benign retroperitoneal schwannomas with bone destruction are often mistaken for malignant neoplasms because correct preoperative diagnosis is difficult. With the lack of specific clinical and radiological characteristics and conclusive preoperative biopsies, the understanding of this unique clinical entity becomes the best diagnostic tool. The lesions are best managed by a meticulously planned total tumor resection surgery with a multidisciplinary team, while maintaining the vascular and neural integrity and spinal stability, in accordance with our proposed management scheme.

Acknowledgment. We would like to express our gratitude to Dr. Abdullrahman Alghamdi from Radiology Department for reviewing the radiologic findings. We would like to thank Editage (www.editage .com) for English language editing.

\section{References}

1. Chiang ER, Chang MC, Chen TH. Giant retroperitoneal schwannoma from the fifth lumbar nerve root with vertebral body osteolysis: a case report and literature review. Arch Orthop Trauma Surg 2009; 129: 495-499.

2. Li Q, Gao C, Juzi JT, Hao X. Analysis of 82 cases of retroperitoneal schwannoma. ANZ J Surg 2007; 77: 237-240.

3. Sakalauskaite M, Stanaitis J, Cepkus S, Pleckaitis M, Lunevicius R. Retroperitoneal giant schwannoma eroding lumbal vertebra: A case report with a literature review. Cent Eur J Med 2008; 3: 233.

4. D'Andrea G, Sessa G, Picotti V, Raco A. One-Step Posterior and Anterior Combined Approach for L5 Retroperitoneal Schwannoma Eroding a Lumbar Vertebra. Case Rep Surg 2016; 2016: 1876765.

5. Kotil K. An extremely giant lumbar schwannoma: new classification (kotil) and mini-open microsurgical resection. Asian Spine J 2014; 8: 506-511.

6. Baten E, Lerut J, Kempeneers I. Hybrid open/closed resection procedure for ancient retroperitoneal Schwannoma: case report and review of the literature. Acta Chir Belg 2016; 116: 289-292. 